\title{
Neurogenesis and brain injury: managing a renewable resource for repair
}

\author{
Anna F. Hallbergson, Carmen Gnatenco, and Daniel A. Peterson \\ Neural Repair and Neurogenesis Laboratory, Department of Neuroscience, The Chicago Medical School, \\ North Chicago, Illinois, USA
}

The brain shows limited ability to repair itself, but neurogenesis in certain areas of the adult brain suggests that neural stem cells may be used for structural brain repair. It will be necessary to understand how neurogenesis in the adult brain is regulated to develop strategies that harness neural stem cells for therapeutic use.

J. Clin. Invest. 112:1128-1133 (2003). doi:10.1172/JCI200320098.

In adult centres the nerve paths are something fixed, ended, immutable. Everything may die, nothing may be regenerated. It is for the science of the future to change, if possible, this harsh decree.

$-\mathrm{S}$. Ramon y Cajal, Degeneration and regeneration of the nervous system, 1928 (1)

\section{Updating Cajal's decree}

As observed by the pioneering neuroscientist Santiago Ramon y Cajal, the mature CNS was distinguished from the developing nervous system by the lack of growth and cellular regeneration. The fixed neuronal population of the adult brain was understood to be necessary to maintain the functional stability of adult brain circuitry. This explanation has also been offered to account for the lack of endogenous CNS repair following injury or disease.

Ever perceptive, Cajal left open the possibility for future advances to alter this "harsh decree," and in the last several decades, mounting evidence has led to the view of the CNS as a dynamic, plastic organ, endowed with some potential for self-repair and regeneration. Recent progress in understanding continued neurogenesis in the adult brain has raised hopes that selfrenewal leading to structural repair by new neurons may even be possible in the mature CNS. Nevertheless, under normal conditions, neurogenesis in the adult brain appears to be restricted to the discrete germinal centers: the subventricular zone and the hippocampal dentate gyrus (2) (Figure 1). While some reports indicate that neurogenesis in the adult CNS may be more

Address correspondence to: Daniel A. Peterson, Neural Repair and Neurogenesis Laboratory, Department of Neuroscience, The Chicago Medical School, 3333 Green Bay Road, North Chicago, Illinois 60064, USA. Phone: (847) 578-3411; Fax: (847) 578-8545; E-mail: daniel.peterson@finchcms.edu.

Conflict of interest: The authors have declared that no conflict of interest exists. widespread and include the cerebral cortex $(3,4)$, other reports cast doubt on these observations (5-7). More study is needed to establish the origin, extent, survival, and function of new neurons in these other regions. This Spotlight summarizes our current understanding of the regulation of adult neurogenesis and its relevance to structural brain repair. We propose strategies for harnessing the potential of neural stem cells for brain repair and consider how to apply these strategies to the aging brain.

\section{Plasticity in adult neurogenesis}

Neurogenesis in the adult brain can be divided into three phases in accordance with the sequence of neurogenesis during CNS development: (a) proliferation, when new cells are generated; (b) migration toward target areas; and (c) terminal differentiation into distinct phenotypes (Figure 1). The use of the term "neurogenesis" implies progression through differentiation and should not be used in cases where only proliferation is studied. It is not yet fully known whether these phases in adult neurogenesis are regulated by the same mechanisms that regulate development, or even whether the same mechanisms regulate neurogenesis in the two adult germinal centers. Nevertheless, new, functional neurons are generated in these areas, expanding the definition of plasticity in the adult brain to now include cellular addition to circuitry (8). New hippocampal neurons may participate in the processing of memory in the hippocampus (9), while new olfactory bulb neurons appear to participate in processing olfactory input (10). Newly generated hippocampal neurons may also contribute to the response of the nervous system to antidepressant administration (11).

Interestingly, adult neurogenesis is not static, but its rate may fluctuate in response to environmental change, even subtle macroenvironmental alterations (2). Examples of positive regulators of neurogenesis 

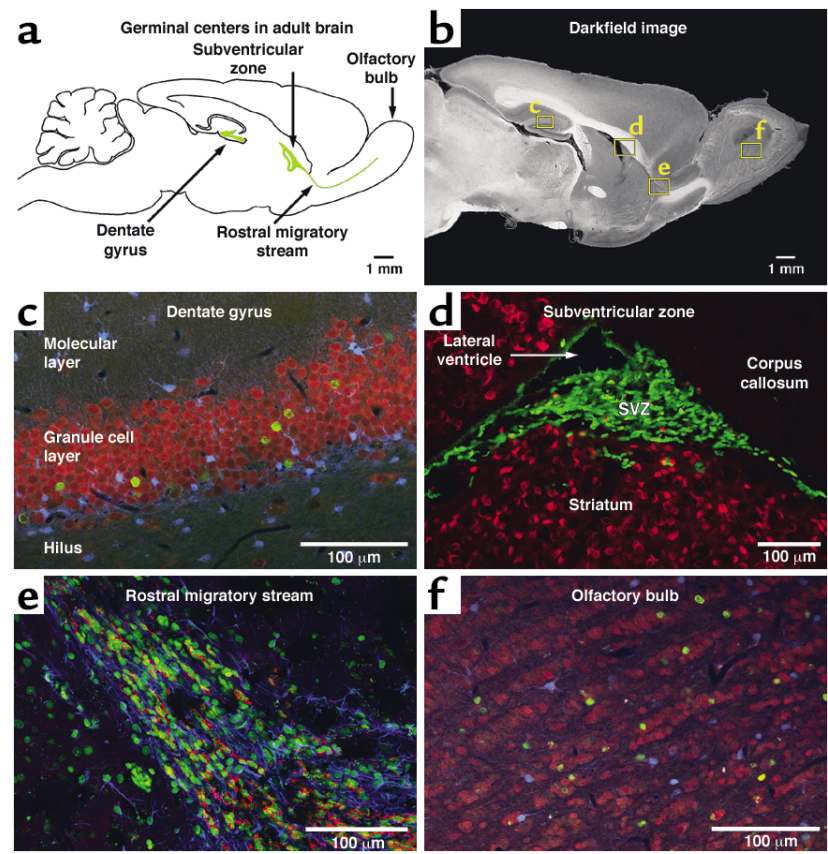

Figure 1

Germinal centers in the adult brain. Neurogenesis in the adult brain is largely confined to two germinal centers: the dentate gyrus and the subventricular zone, shown schematically (a) and in a corresponding sagittal section of the rodent brain (b). Insets in $\mathbf{b}$ show the position of high-resolution micrographs in c-f. In the dentate gyrus (c), newly generated cells are detected through incorporation of the thymidine analog BrdU and labeled with a green fluorophore (Cy2). These cells differentiate into mature neurons, as seen by their coexpression of the marker NeuN (red) but not $\mathrm{S} 100 \beta$ (blue), a marker for mature astrocytes. In contrast, cells generated in the subventricular zone (d) do not differentiate into mature neurons (red) but migrate away through the rostral migratory stream (RMS). Within the RMS (e), newly generated cells are surrounded by astrocytes (glial fibrillary acidic protein [GFAP], blue) and begin to express immature neuronal markers (polysialylated neural cell adhesion molecule [PSA-NCAM], red) as they migrate to the olfactory bulb. Upon arrival in the olfactory bulb (f), newly generated cells differentiate into mature neurons (NeuN, red), but not astrocytes (S100 $\beta$, blue). include physical activity, environmental enrichment, caloric restriction, and modulation of neural activity (12). The responsiveness of adult neurogenesis to environmental influences suggests that its regulation may be under the control of expressed factors whose level of availability dictates the rate of neurogenesis. In normal development, a vast repertoire of proneural genes guide stem cells to a neural fate, and these may continue to be expressed or repressed in neurogenic regions of the adult brain in response to stimuli. Normal CNS development is also guided by the spatial and temporal expression of various trophic or growth factors that guide cell-fate choices and determine the size of the neuronal population. Indeed, the elevated expression of these factors observed in the mature brain following injury has been thought to represent the brain's attempt to protect injured neurons by activating developmental programs (13).

In addition to protecting neurons, trophic factors have been shown to stimulate proliferation of adult-derived neural stem cells and to instruct their differentiation (14-18). For example, FGF-2 is a potent mitogen for a variety of cells and modulates embryonic development and differentiation, adult angiogenesis, wound healing, and tissue repair. In the adult brain, FGF-2 is a survival factor and is neuroprotective against a variety of insults (13). While the basal rate of neurogenesis is the same in wild-type and FGF-2-null mutant mice, Yoshimura et al. (19) found that gene delivery of FGF-2 to the null mutant mice produced an elevation in neurogenesis. This observation demonstrates that neurogenesis is not simply a cell-autonomous property of resident stem cells but is determined by the environmental milieu. Their present study, appearing in this issue of the JCI (20), extends this work by showing that enhanced expression of exogenous
FGF-2 by gene delivery after injury attenuated hippocampal cell loss following lesion while simultaneously upregulating neurogenesis in this region.

\section{Limitations of self-repair by neurogenesis}

Various injury models produce increased neurogenesis in germinal centers (Figure 1), but these regions represent a small portion of the total CNS. What are the prospects for self-repair following injury in other brain structures? As discussed above, some cortical regions may contain newly generated neurons, but these appear to be limited, transient populations, and their confirmation awaits further study $(5,6)$. Most studies using widespread brain injury models, such as ischemic or traumatic brain injury, have reported neurogenesis only within the germinal structures and not in cortical or striatal structures. Even when neurogenesis in the striatum (but not cortex) has been reported following ischemic injury, the resulting neurons survive only a short time (21). Thus, while a growing number of studies report injury-induced enhancement of neurogenesis, it is clear that under normal circumstances such responses do not lead to complete structural or functional recovery in non-neurogenic brain regions.

As in development, the specific temporal and spatial expression of appropriate trophic factors may be necessary not only to achieve the initial generation of new neural progenitor cells, but also to direct their migration, differentiation, and survival. Functional integration of new cells into existing circuitry may require additional signals. In the adult brain, these signals may normally exist only in germinal centers. By performing a targeted cortical lesion, Magavi et al. (22) have shown that progenitor cells can be recruited into the adult cerebral cortex, suggesting that this environment is not 


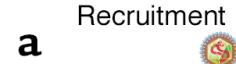

a

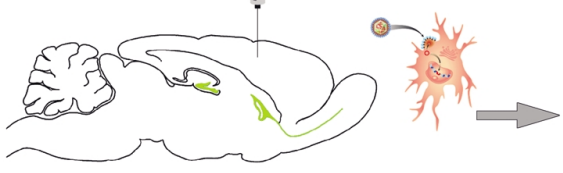
Viral delivery to express
environmental signals

Migration and differentiation of endogenous neural stem cells

\section{b}

Replacement

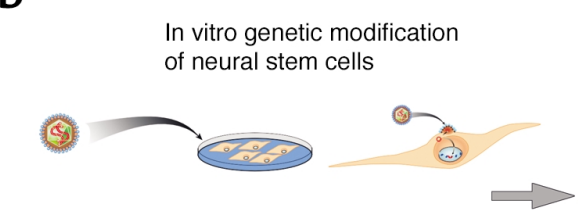

c
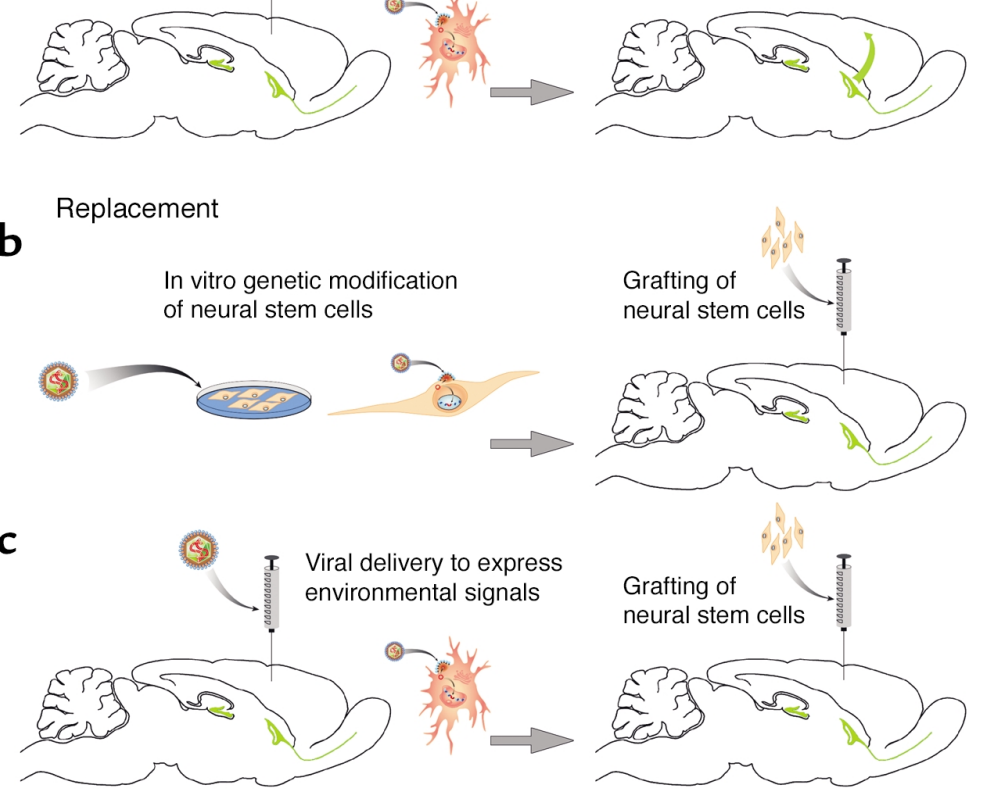

\section{Figure 2}

Therapeutic strategies for brain repair by stem cells. For brain repair in regions outside of the germinal centers, such as the cerebral cortex, stem cells may contribute to repair through recruitment or replacement. In the case of recruitment (a), the environment in the non-neurogenic region must be enhanced with appropriate environmental signals to attract endogenous neural stem cells, possibly from the germinal centers, to expand this population of cells, and to instruct their differentiation into appropriate neurons. This environmental enhancement could most likely be achieved using in vivo gene therapy leading to transgene expression by endogenous cells and may require a precise temporal and spatial delivery of appropriate transgenes to achieve the desired outcome. In the case of replacement (b), neural stem cells derived from embryonic, fetal, or adult sources could be expanded in vitro, directed down specific neuronal lineages, and genetically modified to express the necessary environmental signals. Thus committed to the correct phenotype and expressing necessary environmental signals to ensure their survival, these cells could then be grafted to the target region. Alternatively (c), the environment could be enhanced by gene delivery before (or possibly after) the grafting of the replacement neural stem cells. intrinsically inhibitory to neuronal differentiation. The identification and expression of appropriate signals may lead to structural repair in brain structures other than the germinal centers (23).

\section{Therapeutic prospects for structural brain repair}

Although much has been learned from observing endogenous responses to injuries, it will be necessary to develop targeted therapeutic intervention for brain injury or disease. At the present time, there are two possible therapeutic strategies to achieve structural brain repair: recruitment and replacement (Figure 2).

\section{Recruitment strategies}

Recruitment of endogenous progenitors presents the most elegant strategy for replacing neurons lost to injury or disease. Proliferation of neural progenitors in germinal centers can be induced by delivery of mitogenic trophic factors, such as FGF-2 (2, 20, 24). Recruitment of neural progenitors to adjacent brain structures has also been achieved through delivery of other trophic factors, such as brain-derived neurotrophic factor (BDNF), that may instruct cell differentiation $(16,17)$. By delivering another factor, TGF- $\alpha$, into the striatum, Fallon et al. (25) were able to induce proliferation in the adjacent subventricular zone with subsequent migration of these newly generated cells into the striatum. However, these cells only differentiated following striatal injury, suggesting that additional, unknown signals were required. Recruitment of peripherally derived progenitor cells, such as bloodborne progenitor cells, may provide another cell population for brain repair.
While studies such as those discussed above have generated considerable enthusiasm for the eventual recruitment of endogenous progenitor cells as a therapeutic strategy, the successful development of stem cell recruitment therapy will depend on our ability to manage the proliferation, migration, differentiation, and functional integration of recruited cells. The first step toward this goal is to investigate the regulation of the events that specify cell fate both in cell culture and in vivo. For our understanding to advance, it is critical that a wide range of cell types be studied, from embryonic stem cells to adult-derived cells, to test the generality of the regulatory mechanisms. However, characterizing stem cells will not be sufficient for the development of therapeutic strategies. It will also be necessary to understand how the brain microenvironment changes as a result of injury, disease, and aging, in order to develop therapies that can recruit endogenous progenitors for structural brain repair.

Stem cell recruitment therapy will require targeted and regulated delivery of the key environmental signals or factors to the brain region where repair is needed (Figure 2). One possible route of delivery is the systemic administration of an identified factor or its synthetic precursor. This delivery approach requires that the factor cross the blood-brain barrier and reach the target region at the necessary dose. As factors delivered this way may act upon a wide variety of stem cells, this approach will likely be limited by the nonspecific activation of stem cells in other organ systems or even brain regions other than the intended target. An alternative strategy is the direct, targeted delivery of the factor by intracranial infusion. While this 
approach is suitable for experimental studies, chronic intracranial cannulation of patients will likely present unacceptable health risks. Perhaps the most promising delivery strategy for managing stem cell recruitment is gene therapy (26). While still requiring intracranial injection into the target region, development of viral vectors that can infect postmitotic cells (such as neurons) and that maintain stable, long-term gene expression offer the possibility of requiring only a single delivery episode. With continued advances in regulation of gene expression, it may be possible to turn transgene expression on during the appropriate time frame, or to shut it down entirely when recruitment is not desired. By expressing the transgene directly within the targeted cell, it is possible to extend management of therapeutic factors to directly modify the cell through, for example, the expression of intracellular signals or surface receptors. Finally, it may be possible to construct viral-delivery systems that allow for sequential or simultaneous expression of different environmental signals to accommodate the need to guide recruited stem cells through several stages of cell-fate specification.

\section{Replacement strategies}

Even if all of the key environmental signals necessary to recruit endogenous progenitor cells were determined, the migratory distance from germinal centers would still present an obstacle for repopulation of some brain regions by recruitment. Another challenge to a recruitment strategy is that the different signals needed to achieve migration followed by differentiation into the desired neuronal phenotype may require distinct spatial and temporal delivery of factors that may be difficult to achieve. In such cases, an alternative strategy (Figure 2) is to generate a population of defined progenitor cells in culture that could be tailored to a specific neuronal lineage. These cells would then be grafted into the injured brain region (27). Such cell-replacement strategies have been used for some time, with success primarily in experimental models of Parkinson and Huntington diseases (28). Replacement of lost or injured neurons may be successful where the environment can specify the appropriate fate of the grafted progenitor cells. This is true for progenitor cells grafted into germinal centers but not necessarily for other brain regions (29). While neonatal brains can readily incorporate grafted progenitor cells, relatively few graft-derived neurons are typically found following intrastriatal or intracortical grafting to the mature brain. These results suggest that, in the adult brain, non-neurogenic environments may support only limited differentiation (28). The state of cell differentiation may also be an important factor, as improved survival with intrastriatal transplantation has been obtained using undifferentiated embryonic stem cells (30). Thus success with cell-replacement strategies for structural brain repair may require an appropriate combination of choosing the correct cell-lineage state and creating a supportive brain environment.

The goal of creating a supportive environment for the grafting of replacement cells will likely use the same strategies for trophic factor delivery that are discussed above for recruitment of endogenous cells. In developing therapies, it will be important to understand the brain's response to trophic factor delivery, irrespective of whether this delivery is intended for recruitment or replacement strategies. For example, in studies aimed at preparing the cortical environment for subsequent stem cell grafts, we evaluated the response of the entorhinal cortex to gene delivery of a trophic factor, FGF-2. Lying adjacent to the hippocampus, the entorhinal cortex is important for processing learning and memory, and it suffers neuronal loss early in Alzheimer disease. Normally, there is limited endogenous proliferation in the entorhinal cortex, but delivery of a trophic factor gene, FGF-2, caused an increase in local cortical proliferation (Figure 3) (A.F. Hallbergson and D.A. Peterson, unpublished observations). Similar proliferative responses are found in other cortical regions following trophic factor gene delivery (31). It remains to be determined whether the newly generated endogenous cells are transitory, or whether they survive and differentiate. These observations illustrate that, in the grafting of stem cells to the brain, it may be difficult to discriminate the relative contributions of the specific environmental manipulation (i.e., trophic factor delivery) and the local cellular response (proliferation of endogenous cells) to the survival and incorporation of the grafted stem cells.

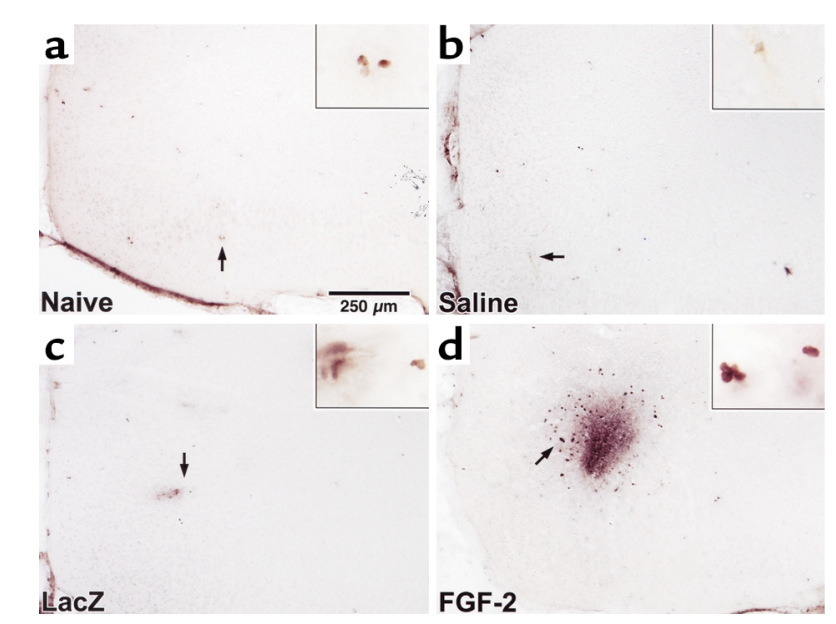

Figure 3

Endogenous cortical proliferation is enhanced following FGF-2 gene delivery. (a) Endogenous proliferation occurs infrequently in the naive entorhinal cortex. Newly generated cells labeled by BrdU administration over 48 hours are indicated by the arrow and shown at higher magnification in the inset. (b) Intracerebral saline injection prior to BrdU treatment produced no visible increase in proliferation in the entorhinal cortex. (c) Injection of adenovirus expressing the reporter gene LacZ produced little effect on proliferation in the entorhinal cortex. (d) Adenovirus-mediated gene delivery of FGF-2 produced a substantially increased proliferation in the entorhinal cortex. 


\section{Structural repair in the aging brain}

Most animal studies investigating the regulation of neurogenesis and the grafting of neural stem cells have been conducted on neonatal or young adult brains, and much of this work has used injury models that approximate neurodegenerative diseases, such as Alzheimer or Parkinson disease or stroke. However, the patient populations targeted by these experiments are older, typically at or beyond the sixth decade of life. Does the aging brain provide the same environment for neurogenesis as does the young adult brain? In aged rodents, there are significantly fewer new neurons generated in the germinal centers $(32,33)$. However, this age-related decline can be reversed, at least in the hippocampus, by environmental enrichment (34), $N$-methyl-D-aspartate receptor antagonist treatment (35), or reduction of corticosteroid levels (36). Reduction of corticosteroid levels may not be a useful therapeutic approach, as lowering levels also leads to neuronal cell death.

The reversal of age-related decline in neurogenesis suggests that there is no intrinsic limitation with aging, and that, instead, the environment may change to provide less support for neurogenesis with aging. One possibility is that the age-related decline in neurogenesis results from reduced trophic factor levels. This possibility is supported by the reported enhancement of neurogenesis in aged rodent brains following exogenous delivery of IGF-I (37), or of FGF-2 or heparinbinding EGF-like growth factor (38). Furthermore, pretreatment with trophic factors improved the survival of fetal neurons grafted to the aged hippocampus $(39,40)$, suggesting that the availability of trophic factors in the aged hippocampus may be limited.

An age-related limitation in the brain environment is also suggested by the clinical experience with fetal-tissue grafts to the brains of Parkinson patients. Freed et al. (41) found that the prospects for clinical improvement decrease as a function of patient age and that fetal transplants may contribute to the development of persistent dyskinesia. Another recent report found no improvement in patient outcome following fetal-neuron transplantation to Parkinson patients (42). Thus, stem cell therapies for the aged brain may hold great clinical potential, but the capacity of the aged brain to support the recruitment or survival of transplanted stem cells remains to be determined. As discussed above, it is likely that the environment of the aged brain will need to be augmented, and investigation into regulation of the capacity of the aged brain to support structural brain repair should receive high priority.

\section{Conclusions}

It is possible in certain circumstances to recruit neural stem cells in the adult brain to contribute new neurons into areas depleted by experimental injury. Much work is required to elucidate the signals involved in recruiting stem cells for this purpose. Not only must environmental changes resulting from disease pathology or injury response be better understood, but the capacity of undifferentiated stem cells to respond to the spatial and temporal presentation of these signals must be determined. The prospects for developing therapeutic applications from advancing our understanding of how stem cell fate is managed relies on continued and unfettered basic research into fundamental properties of embryonic and adult-derived stem cells. Finally, agerelated changes in the brain's capacity to support both endogenous and grafted neural stem cells need to be better understood to advance the possible harnessing of neurogenesis for structural brain repair.

1. Ramon y Cajal, S. 1928. Degeneration and regeneration of the nervous system. Volume 2. Haffner Publishing Co. New York, New York, USA. p. 750. 2. Peterson, D.A. 2002. Stem cells in brain plasticity and repair. Curr. Opin. Pharmacol. 2:34-42.

3. Gould, E., Vail, N., Wagers, M., and Gross, C.G. 2001. Adult-generated hippocampal and neocortical neurons in macaques have a transient existence. Proc. Natl. Acad. Sci. U. S. A. 98:10910-10917.

4. Lie, D.C., et al. 2002. The adult substantia nigra contains progenitor cells with neurogenic potential. J. Neurosci. 22:6639-6649.

5. Kornack, D.R., and Rakic, P. 2001. Cell proliferation without neurogenesis in adult primate neocortex. Science. 294:2127-2130.

6. Koketsu, D., Mikami, A., Miyamoto, Y., and Hisatsune, T. 2003. Nonrenewal of neurons in the cerebral neocortex of adult macaque monkeys. J. Neurosci. 23:937-942.

7. Ehninger, D., and Kempermann, G. 2003. Regional effects of wheel running and environmental enrichment on cell genesis and microglia proliferation in the adult murine neocortex. Cereb. Cortex. 13:845-851.

8. van Praag, H., et al. 2002. Functional neurogenesis in the adult hippocampus. Nature. 415:1030-1034.

9. Shors, T.J., et al. 2001. Neurogenesis in the adult is involved in the formation of trace memories. Nature. 410:372-376.

10. Carleton, A., Petreanu, L.T., Lansford, R., Alvarez-Buylla, A., and Lledo, P.M. 2003. Becoming a new neuron in the adult olfactory bulb. Nat. Neurosci. 6:507-518.

11. Santarelli, L., et al. 2003. Requirement of hippocampal neurogenesis for the behavioral effects of antidepressants. Science. 301:805-809.

12. Ray, J., and Peterson, D.A. 2002. Neural stem cells in the adult hippocampus. In Neural stem cells for brain and spinal cord repair. T. Zigova, E.Y. Snyder, and P.R. Sanberg, editors. Humana Press. Totawa, New Jersey, USA. 269-286.

13. Peterson, D.A., and Gage, F.H. 1999. Trophic factor therapy for neuronal death. In Alzheimer disease. R.D. Terry, R. Katzman, K.L. Bick, and S.S. Sisodia, editors. Lippincott-Raven Publishers. Philadelphia, Pennsylvania, USA. 373-388.

14. Kuhn, H.G., Winkler, J., Kempermann, G., Thal, L.J., and Gage, F.H. 1997. Epidermal growth factor and fibroblast growth factor-2 have different effects on neural progenitors in the adult rat brain. J. Neurosi. 17:5820-5829.

15. Zigova, T., Pencea, V., Wiegand, S.J., and Luskin, M.B. 1998. Intraventricular administration of BDNF increases the number of newly generated neurons in the adult olfactory bulb. Mol. Cell. Neurosci. 11:234-245.

16. Pencea, V., Bingaman, K.D., Wiegand, S.J., and Luskin, M.B. 2001. Infusion of brain-derived neurotrophic factor into the lateral ventricle of the adult rat leads to new neurons in the parenchyma of the striatum, septum, thalamus, and hypothalamus. J. Neurosci. 21:6706-6717.

17. Benraiss, A., Chmielnicki, E., Lerner, K., Roh, D., and Goldman, S.A. 2001. Adenoviral brain-derived neurotrophic factor induces both neostriatal and olfactory neuronal recruitment from endogenous progenitor cells in the adult forebrain. J. Neurosci. 21:6718-6731.

18. Aberg, M.A., Aberg, N.D., Hedbacker, H., Oscarsson, J., and Eriksson, P.S. 2000. Peripheral infusion of IGF-I selectively induces neurogenesis in the adult rat hippocampus. J. Neurosci. 20:2896-2903.

19. Yoshimura, S., et al. 2001. FGF-2 regulation of neurogenesis in adult hippocampus after brain injury. Proc. Natl. Acad. Sci. U. S. A. 98:5874-5879.

20. Yoshimura, S., et al. 2003. FGF-2 regulates neurogenesis and degeneration in the dentate gyrus after traumatic brain injury in mice. J. Clin. Invest. 112:1218-1226. doi:10.1172/JCI200316618.

21. Arvidsson, A., Collin, T., Kirik, D., Kokaia, Z., and Lindvall, O. 2002. Neuronal replacement from endogenous precursors in the adult brain after stroke. Nat. Med. 8:963-970.

22. Magavi, S.S., Leavitt, B.R., and Macklis, J.D. 2000. Induction of neurogenesis in the neocortex of adult mice. Nature. 405:951-955.

23. Arlotta, P., Magavi, S.S., and Macklis, J.D. 2003. Molecular manipulation of neural precursors in situ: induction of adult cortical neuro- 
genesis. Exp. Gerontol. 38:173-182.

24. Nakatomi, H., et al. 2002. Regeneration of hippocampal pyramidal neurons after ischemic brain injury by recruitment of endogenous neural progenitors. Cell. 110:429-441.

25. Fallon, J., et al. 2000. In vivo induction of massive proliferation, directed migration, and differentiation of neural cells in the adult mammalian brain. Proc. Natl. Acad. Sci. U. S. A. 97:14686-14691.

26. Peterson, D.A., Ray, J., and Gage, F.H. 2000. Future prospects of gene therapy for treating CNS diseases. In Innovative animal models of CNS diseases: from molecule to therapy. D.F. Emrich, R.L. Dean, and P.R. Sanberg, editors. Humana Press. Totawa, New Jersey, USA. 378-388.

27. Wu, P., et al. 2002. Region-specific generation of cholinergic neurons from fetal human neural stem cells grafted in adult rat. Nat. Neurosci. 5:1271-1278.

28. Bjorklund, A., and Lindvall, O. 2000. Cell replacement therapies for central nervous system disorders. Nat. Neurosci. 3:537-544.

29. Suhonen, J.O., Peterson, D.A., Ray, J., and Gage, F.H. 1996. Differentiation of adult hippocampus-derived progenitors into olfactory neurons in vivo. Nature. 383:624-627.

30. Bjorklund, L.M., et al. 2002. Embryonic stem cells develop into functional dopaminergic neurons after transplantation in a Parkinson rat model. Proc. Natl. Acad. Sci. U. S. A. 99:2344-2349.

31. Hallbergson, A.F., Vega, C.J., Peterson, L.D., and Peterson, D.A. 2003. In vivo gene delivery of FGF-2 stimulates proliferation in the adult cerebral cortex: implications for therapeutic recruitment of neural progenitor cells. Proceedings of the Keystone Symposium: from stem cells to therapy. Steamboat Springs, Colorado, USA. E3:3025. (Abstr.)

32. Kuhn, H.G., Dickinson-Anson, H., and Gage, F.H. 1996. Neurogenesis in the dentate gyrus of the adult rat: age-related decrease of neuronal progenitor proliferation. J. Neurosci. 16:2027-2033.
33. Peterson, L.D., and Peterson, D.A. 2002. Age-related environmental impairment of olfactory bulb neurogenesis. Proceedings of the Federation of European Neuroscience Societies. 3:69.10. (Abstr.)

34. Kempermann, G., Kuhn, H.G., and Gage, F.H. 1998. Experienceinduced neurogenesis in the senescent dentate gyrus. J. Neurosci. 18:3206-3212.

35. Nacher, J., Alonso-Llosa, G., Rosell, D.R., and McEwen, B.S. 2003 NMDA receptor antagonist treatment increases the production of new neurons in the aged rat hippocampus. Neurobiol. Aging. 24:273-284.

36. Cameron, H.A., and McKay, R.D. 1999. Restoring production of hippocampal neurons in old age. Nat. Neurosci. 2:894-897.

37. Lichtenwalner, R.J., et al. 2001. Intracerebroventricular infusion of insulin-like growth factor-I ameliorates the age-related decline in hippocampal neurogenesis. Neuroscience. 107:603-613.

38. Jin, K., et al. 2003. Neurogenesis and aging: FGF-2 and HB-EGF restore neurogenesis in hippocampus and subventricular zone of aged mice. Aging Cell. 2:175-183.

39. Zaman, V., and Shetty, A.K. 2002. Combined neurotrophic supplementation and caspase inhibition enhances survival of fetal hippocampal CA3 cell grafts in lesioned CA3 region of the aging hippocampus. Neuroscience. 109:537-553.

40. Zaman, V., and Shetty, A.K. 2003. Fetal hippocampal CA3 cell grafts enriched with fibroblast growth factor-2 exhibit enhanced neuronal integration into the lesioned aging rat hippocampus in a kainate model of temporal lobe epilepsy. Hippocampus. 13:618-632.

41. Freed, C.R., et al. 2001. Transplantation of embryonic dopamine neurons for severe Parkinson's disease. N. Engl. J. Med. 344:710-719.

42. Olanow, C.W., et al. 2003. A double-blind controlled trial of bilateral fetal nigral transplantation in Parkinson's disease. Ann. Neurol. 54:403-414. 